\title{
The Outpatient Parenteral Antimicrobial Therapy (OPAT) Experience in a Referral Hospital in South Carolina
}

\author{
Julie Coursen ${ }^{1}$, Prerana Roth ${ }^{2,}$, , Christopher Schrank ${ }^{2}$, John Schrank ${ }^{2}$ \\ ${ }^{1}$ Department of Medicine, University of South Carolina School of Medicine Greenville, Greenville, USA \\ ${ }^{2}$ Department of Medicine, Prisma Health, Greenville, USA
}

Email address:

Julie.coursen@gmail.com (J. Coursen), Prerana.Roth2@prismahealth.org (P. Roth)

${ }^{*}$ Corresponding author

\section{To cite this article:}

Julie Coursen, Prerana Roth, Christopher Schrank, John Schrank. The Outpatient Parenteral Antimicrobial Therapy (OPAT) Experience in a Referral Hospital in South Carolina. International Journal of Infectious Diseases and Therapy. Vol. 5, No. 4, 2020, pp. $112-117$. doi: $10.11648 /$ j.ijidt.20200504.12

Received: September 20, 2020; Accepted: September 29, 2020; Published: October 13, 2020

\begin{abstract}
Several studies have established outpatient parenteral antibiotic therapy (OPAT) as an alternative to prolonged inpatient stays to reduce healthcare expenditure, decrease hospital admission times, and increase patient satisfaction. However, studies have also shown significant adverse events occurring while receiving treatment outpatient. We collected retrospective data through electronic medical record review on all patients discharged on IV antibiotics whose OPAT was managed by the infectious disease specialists at Greenville Health System between 1/1/17 and 6/30/17. There were a total of 336 individual patients discharged on OPAT during the 6 month period. Bacteremia (25.4\%), osteomyelitis (14.9\%), and diabetic foot infections $(12.8 \%)$ were the most common indications for OPAT with methicillin-sensitive staphylococcus aureus (MSSA) being the most common organism targeted $(22.5 \%) .11 \%$ of patients had a medication change during their treatment course. The most common reasons were nausea/malaise $(26 \%)$ and acute kidney injury $(26 \%)$. Our hospital re-admission rate was $8.7 \%$. Statistical analysis of the data indicated that home infusion was significantly more likely to result in re-admission compared to the infusion center $(\mathrm{p}=0.02)$. Also receiving antibiotics for osteomyelitis was more likely to result in re-admission compared to other diagnoses $(\mathrm{p}=0.048)$. Our data indicates that self-administration of antibiotics at home results in higher readmission rates compared to administration at infusion centers. Factors that may contribute to this difference such as compliance, co-morbidities, or frequency of nurse assessments warrant further exploration to optimize the safety of OPAT, especially in rural South Carolina.
\end{abstract}

Keywords: Outpatient Parenteral Antimicrobial Therapy (OPAT), Infusion Center, Rural Home Care, Readmission

\section{Introduction}

Outpatient parenteral antimicrobial therapy (OPAT) allows patients to receive intravenous antibiotics for the treatment of infectious diseases outside of the inpatient hospital setting. First described as a treatment option in the United States in 1974, studies have since established OPAT as an alternative to reduce healthcare expenditure, decrease hospital admission times, and increase patient satisfaction [1, 2, 8, 14]. Outpatient administration allows for reduced exposure to nosocomial infections, and rates of Clostridium difficile infection in OPAT have been as low as $0.1 \%$ [14]. OPAT has been used effectively for the treatment of many infectious diseases, including bone and joint infections, skin infections, and endocarditis [3-6]. OPAT has also demonstrated efficacy in higher risk patients, including the elderly and those with prosthetic valves $[6,7]$. Clinical success rates are estimated up to $99 \%$, and mortality rates are generally low, ranging from $0 \%$ to $1.4 \%[12,23]$. Patients are selected for OPAT based on their medical stability, the clinical course of their infection, reimbursement status, and their anticipated adherence to an outpatient program [8, 24]. Patients may receive antibiotics at home or at an infusion center close to their home.

Despite its benefits to both the physician and patient, OPAT poses significant potential risks, including treatment failure, hospital readmissions, adverse drug events, and catheter- 
related complications [13]. Treatment failure is most often due to relapse of the primary infection, with the clear majority of treatment failures occurring within the first year of treatment $[3,8,15,22]$. Many studies have contributed their outcomes to the body of OPAT literature, but rates have varied markedly. For example, reported rates of readmission have ranged from $3.6 \%$ to $27 \%$, and rates of adverse drug reaction have varied from $0.3 \%$ to $30.2 \% \quad[12,14,20]$. Catheter-related complications have been reported at about $3.2-5.3$ per 1000 line days [14].

Many studies have examined risk factors for unfavorable outcomes in OPAT. Comorbidities such as HIV, peripheral vascular disease, and diabetes have been found to increase the chance of treatment failure, and cancer, diabetes, and UTI have been associated with hospital readmission [4, 5, 11, 16, 17, 22]. Hospital readmission has also been associated with drugresistant organisms, previous hospital admission within the last year, and lack of laboratory monitoring $[16,19]$. Studies have also demonstrated variation in outcomes with antimicrobial classes. Notably, vancomycin and daptomycin have been associated with increased adverse drug reactions [20]. Demographics appear to contribute to the success or failure of treatment as well. Older patients and females are at particularly increased risk of adverse drug reactions, hospital readmission, and treatment failure $[5,18,20]$. Primary care coverage is a protective factor, as patients followed by a primary care physician are less likely to be readmitted for hospitalization [16].

Because of the widespread use of OPAT in infectious disease practices today, there is great benefit in better understanding the risks and advantages offered by OPAT. It is particularly valuable to identify which factors increase risk for unfavorable outcomes, so that we might improve patient safety and treatment. Due to our evolving understanding of OPAT outcomes, this study aims to analyze the rates of treatment success, hospital readmissions, adverse drug events, catheter-related complications, and risk/protective associations for patients discharged on OPAT from our hospital which serves as the referral center for much of upstate rural South Carolina.

\section{Methods}

We performed a retrospective chart review of all patients $(n=336)$ that received OPAT under the guidance of the Prisma Health-Upstate Infectious Disease Specialty group over the 6-month period of January 1, 2017 - June 30, 2017. We collected demographic information on age, sex, and insurance status. We collected clinical information including culture result, choice and duration of parenteral antimicrobial therapy, concomitant usage of oral antibiotics, and location of OPAT (home versus infusion center). We excluded patients getting antibiotics with dialysis or at skilled nursing facilities.

Our goal was to identify the factors associated with both successful treatment outcomes and adverse events. Outcomes included treatment completion rates, readmission prior to completion of antibiotic therapy, adverse events such as catheter related events and medication side effects, and relapse of infection which was defined as need for retreatment within 6 months of initial antibiotic course. We also collected data on missed doses, medication changes, reason for medication changes, documented Clostridium difficile infection, and death. A chi-square test of independence was performed to examine the effect of individual variables (for example, antibiotic used or OPAT site) on outcome variables of re-admission and mortality.

\section{Results}

Based on demographic information collected, our patient population was relatively elderly, and we found a larger portion on Medicare/Medicaid compared to previously reported studies [20]. The average age of our patients was 59.5 years, and the patients were $57 \%$ male and $43 \%$ female. $46 \%$ of our patients were insured with Medicare, $14 \%$ with Medicaid, and $15 \%$ had no insurance. $55 \%$ of our patients chose to receive OPAT at an infusion center and $45 \%$ received OPAT at home.

Our most common indication for treatment was osteomyelitis at $28 \%$, divided approximately in half between patients with diabetes and those without. Bacteremia was another significant indication at 25\% (Figure 1). Methicillinsusceptible Staphyloccocus aureus was the most common organism treated. Fortunately, our population has a lower MRSA burden than many densely-populated cities. Ceftriaxone and vancomycin were the most common antibiotics chosen (Figure 2). The average duration of treatment was 4.78 weeks.

Adverse events related to medication were tracked as a measure of clinical outcomes and treatment success. $11 \%$ of patients required a change in medication due to adverse side effects or intolerance. Nausea/malaise and acute kidney injury were the most common reasons at $26 \%$ each. This rate supports the work of previous studies, which have documented a $14 \%$ rate of adverse events requiring medication change [20]. We found an $8.6 \%$ rate of missed medication doses; however, this is likely an underestimate, as this number is limited by infusion documentation or patient self-reporting.

We had very low rates of complications related to treatment. We found a $1.5 \%$ catheter-line associated bloodstream infection. There was a single case of deep vein thrombosis, and three cases of Clostridium difficile infection. We had three cases of death, documented from all-cause mortality. Previous studies have documented a rate of $<1 \%$ of mortality and Clostridium difficile infection, and our results support this claim [14].

We measured treatment success through assessing completion rates, need for retreatment, and hospital readmission rates. Our completion rate was highly successful at $94 \%$, and our need for retreatment due to recurrent infection was low at $7 \%$. Our hospital readmission rate prior to antibiotic completion was $8.7 \%$, which is consistent with previously reported rates ranging from 3.6\%-12.6\% [14]. We noted all-cause hospital readmission, regardless of whether 
the readmission was related to recurrent infection, treatment adverse event, comorbidities, or new acute illness. In sum, our results were consistent with and in support of previously reported clinical outcomes.

A novel finding in our study was the increased risk of hospital readmission in patients who received their therapy at home as opposed to receiving it at an infusion center. Patients who received OPAT through home infusion were more likely to have a hospital readmission compared to patients receiving OPAT at an infusion center $(\mathrm{P}=0.02)$. This relationship is newly found without previous evidence documented in the infectious disease literature to our knowledge.

\section{Discussion}

Based on our retrospective chart review, outcomes of Greenville Health System outpatient parenteral antibiotic therapy are consistent with previously reported rates in the greater clinical community. Additionally, we found a novel association between hospital readmissions and home infusion administration. This finding is an area for future study as this relationship may represent an opportunity for significant clinical improvement. Further investigation would give us the opportunity to identify risk factors for hospital readmission, improve clinical care, and more effectively counsel patients during this decision.

There are several possible factors that may explain the relationship between home infusion and hospital readmission. First, this relationship could possibly be due to administration technique. Patients are regularly discharged with a peripheral inserted central catheter after receiving inpatient education for self-administration or administration by a family member or friend. However, this education is limited, and many patients feel hesitant about administering antibiotics to themselves [25]. Though many papers show that even patients with lower education levels can administer antibiotics to themselves safely; in our experience, some patients may be unwittingly practicing poor technique that puts them at risk for recurrent infection and hospital readmission [9, 10, 21, 26]. For example, several patients do not fully understand nuances such as lines need to be capped or that pets should not be allowed near supplies.

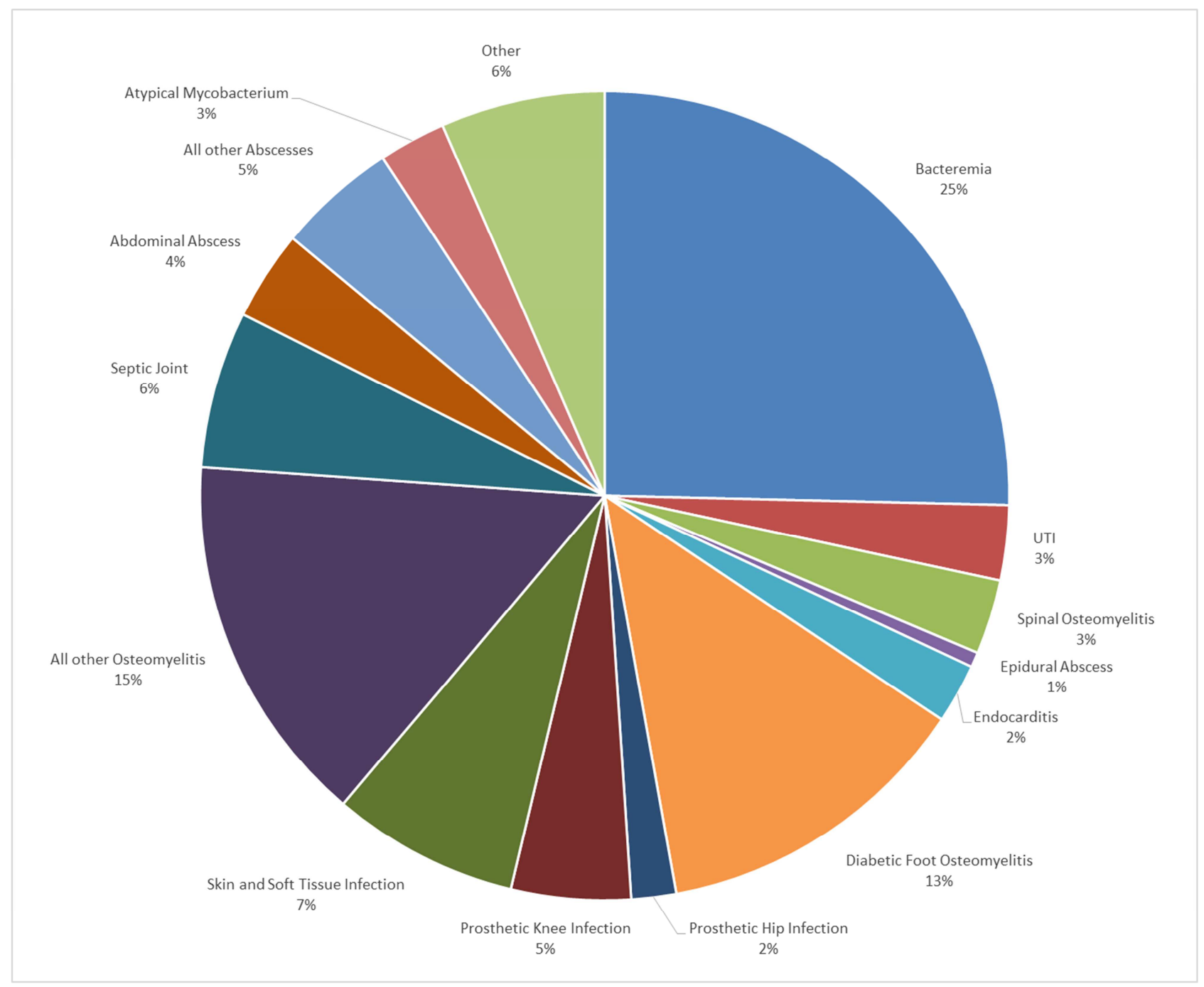

Figure 1. Indication for IV Antibiotics. 


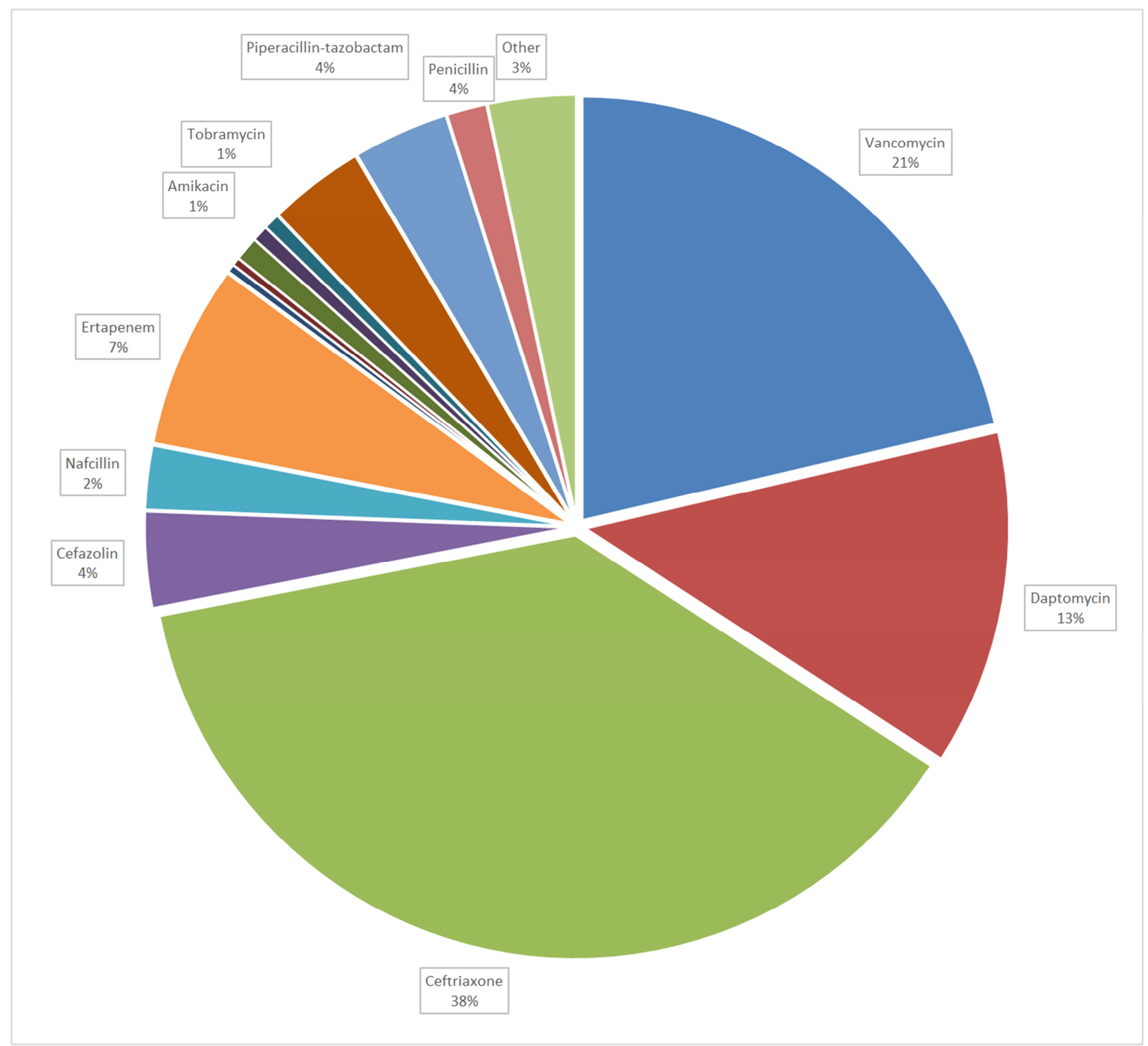

Figure 2. Antibiotic choice for OPAT.

Another possibility from our experience is that patients who choose home infusion may have poor functional status that prohibits them from traveling regularly to an infusion center. For example, patients with super-morbid obesity or those who are wheel-chair bound are more likely to choose home infusion if possible. These comorbidities make them a more debilitated population. Indeed, there were four cases of acute kidney injury that led to hospital readmission, but the majority of these readmissions were not directly related to medication adverse event or recurrent infection. This suggests that comorbidities or other afflictions were driving the hospital readmissions rather than the OPAT itself. Several of our patients had a malignancy alongside their infection, which likely influenced both their risk factors for readmission and their decision for home infusion. Notably, we tracked all-cause hospital readmission, so we did not differentiate between patients who were readmitted due to
OPAT versus other illnesses. Therefore, it is possible that many of the patients receiving home infusion were simply sicker than those receiving OPAT at an infusion center.

Patients receiving OPAT through an infusion center may also have intangible benefits that are less available to patients using home infusion. For example, infusion center administration allows for more interaction with healthcare providers. Through daily visits to the infusion center, it is possible that these patients would receive more thorough line care and more frequent overall clinical examination. There is less opportunity for clinical examination for patients receiving home OPAT, which may put them at greater risk for hospital readmission.

There are some limitations to our study, such as the inherent limitation of retrospective chart review in capturing patient perspective. For example, it would be beneficial to complete a prospective study that allowed for greater patient 
input. Documenting the patient perspective would allow us to better understand the personal factors that patients consider when deciding home versus infusion center. Further, it would provide an opportunity to more closely monitor patients undergoing home infusion with the hopes of eliminating any confounding factors associated with poor technique or compliance. Indeed, many home infusion companies are now able to send a nurse to assess the patient daily.

Because of the broad use of OPAT in practice today, there is great clinical value in better understanding the potential opportunities for improvement throughout OPAT practice. Our study found that home infusion was associated with an increased risk for hospital readmission compared to infusion centers, and understanding this relationship is necessary to provide the best clinical care for our patients. Further investigation into the outcomes of OPAT in relation to treatment location will advance our understanding, improve clinical care, and build on the foundation of excellent patient outcomes through outpatient parenteral antibiotic therapy.

\section{Conclusion}

Our study shows OPAT is associated with high treatment success rates and is an excellent option for the treatment of complex infectious diseases in the outpatient setting. Our hospital readmission rate of $8.7 \%$ was similar to prior studies. Patients who self-administered antibiotics at home were more like to be re-admitted to the hospital than those who received antibiotics at the infusion center $(p=0.02)$. This finding may be related to compliance, administration technique, frequency of medical assessments, underlying comorbidities, or severity of illness. Further research would help optimize patient outcomes and prevent readmissions.

\section{References}

[1] Rucker RW, Harrison GM. Outpatient intravenous medications in the management of cystic fibrosis. Pediatrics 1974; 54: 358-60.

[2] Chapman ALN, Dixon S, Andrews D, Lillie PJ, Bazaz R, Patchett JD. Clinical Efficacy and Cost-Effectiveness of Outpatient Parenteral Antibiotic Therapy: a UK Perspective. Journal of Antimicrobial Chemotherapy. 2009; Vol. 64.

[3] Tice AD. The Use of Outpatient Parenteral Antimicrobial Therapy in the Management of Osteomyelitis: Data from the Outpatient Parenteral Antimicrobial Therapy Outcomes Registries. Chemotherapy. 2001; Vol. 47.

[4] Tice AD, Hoaglund PA, Shoultz DA. Outcomes of Osteomyelitis Among Patients Treated with OPAT. The American Journal of Medicine. 2003; Vol. 114.

[5] Seaton RA, Sharp E, Bezlyak V, Weir CJ. Factors Associated with Outcome and Duration of Therapy in Outpatient Parenteral Antibiotic Therapy (OPAT) Patients with Skin and Soft Tissue Infections. International Journal of Antimicrobial Agents. 2011; Vol. 38.
[6] Partridge DG, O’Brien E, Chapman AL. Outpatient Parenteral Antibiotic Therapy for Infective Endocarditis: A Review of 4 Years' Experience at a UK Centre. Postgraduate Medical Journal. 2012; Vol. 88.

[7] Mujal A, Sola J, Hernandez M, Villarino M, Baylina M, Tajan J, Oristrell J. Safety and Effectiveness of Outpatient Parenteral Antimicrobial Therapy in Older People. Journal of Antimicrobial Chemotherapy. 2016; Vol. 71.

[8] Paladino JA, Poretz D. Outpatient Parenteral Antimicrobial Therapy Today. Clinical Infectious Diseases. 2010; Vol. 51 (Supplemental 2): S198-208.

[9] Matthews PC, Conlon CP, Berendt AR, Kayley J, Jefferies L, Atkins BL, Byren I. Outpatient Parenteral Antimicrobial Therapy: Is it Safe for Patients to Self-Administer at Home? A Retrospective Analysis of a Large Cohort over 13 Years. Journal of Antimicrobial Chemotherapy. 2007; Vol. 60.

[10] Chary A, Tice AD, Martinelli LP, Liedtke LA, Plantenga MS, Strausbaugh LJ. Experience of Infectious Disease Consultants with Outpatient Parenteral Antimicrobial Therapy: Results of an Emerging Infections Network Survey. Clinical Infectious Diseases. 2006; Vol. 43.

[11] Berman SJ, Johnson EW. Out-Patient Parenteral Antibiotic Therapy: Clinical Outcomes and Adverse Effects. Hawaii Medical Journal. 2001; Vol. 60.

[12] Sriskandarajah S, Hobbs J, Roughead E, Ryan M, Reynolds K Safety and Effectiveness of 'Hospital in the Home' and 'Outpatient Parenteral Antimicrobial Therapy' in Different Age Groups: A Systematic Review of Observational Studies." The International Journal of Clinical Practice. 2018.

[13] Hoffman-Terry ML, Fraimow HS, Fox TR, Swift BG, Wolf JE. Adverse Effects of Outpatient Parenteral Antibiotic Therapy. American Journal of Medicine. 1999; Vol. 106.

[14] Mackenzie M, Rae N, Nathwani D. Outcomes from Global Adult Outpatient Parenteral Antimicrobial Therapy Programmes: A Review of the Last Decade. International Journal of Antimicrobial Agents. 2014; Vol. 43.

[15] Mackintosh CL, White HA, Seaton RA. Outpatient Parenteral Antibiotic Therapy for Bone and Joint Infections: Experience from a UK Teaching Hospital. Journal of Antimicrobial Chemotherapy. 2011; Vol. 66.

[16] Means L, Bleasdale S, Sikka M, Gross AE. Predictors of Hospital Readmission in Patients Receiving OPAT. Pharmacotherapy. 2016; Vol. 36.

[17] Petrak RM, Skorodin NC, Fliegelman RM, Hines DW, Chundi VV, Harting BP. Value and Clinical Impact of an Infectious Disease Supervised Outpatient Parenteral Antibiotic Program. Open Forum Infectious Diseases. 2016.

[18] Allison GM, Muldoon EG, Kent DM, Paulus JK, Ruthazer R, Ren A, Syndman DR. Prediction Model for 30-Day Hospital Readmissions Among Patients Discharged Receiving Outpatient Parenteral Therapy. Clinical Infectious Diseases. 2014; Vol. 58.

[19] Huck D, Ginsberg, JP, Gordon, SM, Nowacki AS, Rehm SJ, Shrestha NK. Association of Laboratory Test Result Availability and Rehospitalizations in an Outpatient Parenteral Antimicrobial Therapy Programme. Journal of Antimicrobial Chemotherapy. 2014; Vol. 69. 
[20] Keller SC, Williams D, Gavgani M, Hirsch D, Adamovich J, Hohl D, Gurses AP, Cosgrove SE. Rates of and Risk Factors for Adverse Drug Events in Outpatient Parenteral Antimicrobial Therapy. Clinical Infectious Diseases. 2017; Vol. 20.

[21] Kieran J, O'Reilly A, Parker J, Clarke S, Bergin C. SelfAdministered Outpatient Parenteral Antimicrobial Therapy: A Report of Three Years Experience in the Irish Healthcare Setting. European Journal of Clinical Microbiology and Infectious Diseases. 2009; Vol. 28.

[22] Yan M, Elligsen M, Simor AE, Daneman N. Patient Characteristics and Outcomes of Outpatient Parenteral Antimicrobial Therapy: A Retrospective Study. Canadian Journal of Infectious Diseases and Medical Microbiology. 2016.

[23] Tice AD. Experience with a Physician-Directed, Clinic-Based Program for Outpatient Parenteral Antibiotic Therapy in the USA. European Journal of Clinical Microbiology and Infectious Diseases. 1995. Vol. 14; 655-661.
[24] Tice AD, Rehm SJ, Dalovisio JR, Bradley JS, Martinelli LP, Graham DR, Gainer RB, Kunkel MK, Yancey RW, Williams DN. Practice Guidelines for Outpatient Parenteral Antimicrobial Therapy. IDSA Guidelines. Clinical Infectious Diseases. 2004; Vol 38, 358-60.

[25] Tonna A, Anthony G, Tonna I, et al. Home self-administration of intravenous antibiotics as part of an outpatient parenteral antibiotic therapy service: a qualitative study of the perspectives of patients who do not self-administer. BMJ Open 2019; 9 (1): e027475. Published 2019 Jan 25. doi: 10.1136/bmjopen-2018-027475.

[26] Bhavan KP, Brown LS, Haley RW (2015) Self-Administered Outpatient Antimicrobial Infusion by Uninsured Patients Discharged from a Safety-Net Hospital: A Propensity-ScoreBalanced Retrospective Cohort Study. PLoS Med 12 (12): e1001922. https://doi.org/10.1371/journal.pmed.1001922. 